CLAWAR 2020: 23rd International Conference on

Climbing and Walking Robots and the Support

Technologies for Mobile Machines,

Moscow, Russian Federation, 24-26 August 2020.

https://doi.org/10.13180/clawar.2020.24-26.08.54

\title{
PROPELLERLESS AQUATIC ROBOTS
}

\author{
Anton V. Klekovkin ${ }^{1,2}$, Ivan S. Mamaev ${ }^{1}$, Evgeny V. Vetchanin ${ }^{3}$, Valentin A. Tenenev ${ }^{1}$ and \\ Yury L. Karavaev ${ }^{1,2}$ \\ ${ }^{1}$ Kalashnikov Izhevsk State Technical University, Izhevsk, Russia \\ ${ }^{2}$ Center for Technologies in Robotics and Mechatronics Components, \\ Innopolis University, Innopolis, Russia \\ ${ }^{3}$ Udmurt State University, Izhevsk, Russia \\ E-mail: klanvlad@mail.ru, mamaev@rcd.ru, eugene186@mail.ru,v.tenenev@gmail.com,karavaev \\ yury@istu.ru
}

\begin{abstract}
This paper is devoted to investigations of the motion of the propellerless aquatic robots. There are two models of aquatic robots under consideration that move due to rotation of internal rotors. Mathematical models to describe the motion of the robots are proposed. Experiments with different control actions for fabricated prototypes to verify mathematical models have been conducted.
\end{abstract}

\section{Introduction}

Recently, a lot of effort has gone into investigating autonomous mobile robots with new principles of motion on solid surfaces and in fluid. ${ }^{1-7}$ This motion can be implemented by vibrations or periodic controls. Some studies consider the dynamics of propellerless aquatic robots. The motion of such vehicles is achieved by periodically moving internal masses or by rotating rotors. ${ }^{8-12}$ This method of motion can have advantages in some problems, for example, in working at great depths with high hydrostatic pressure and in aggressive environments, since the body can be made completely waterproof and resistant to external influences. Also, there are problems of investigations and monitoring in a fluid in places that are difficult for propeller vehicles (high density of aquatic plants), with high environmental standards.

This paper considers two models of aquatic robots that move by rotation of internal rotors. Mathematical models of movement for each robot are developed, and results of experimental investigation are described.

\section{Description of the robot design}

Two prototypes of propellerless non-deformable aquatic robots were created: a propellerless underwater robot and a propellerless above-water robot with a sharp edge (see Figure 1). Below we consider the design of each of the robots.

\subsection{Design of the propellerless underwater robot}

The propellerless underwater robot has the shape of an ellipsoid of revolution. The body consists of two semi-shells attached to each other along the equatorial plane. The size of the ellipsoid on the larger axis is $300 \mathrm{~mm}$, on the smaller axis is $200 \mathrm{~mm}$. Three pairs of rotors (one large pair and two small ones) are installed inside the shell. The axes of the rotors are located at an angle of $90^{\circ}$ to each other. This arrangement of rotors allows to get a certain vector of the gyrostatic momentum, which is used as a control action. The rotors are driven by DC gear motors.

There are also two buoyancy modules inside the shell that are used for robot diving. The robot is equipped with two pressure sensors for depth control. To determine the ori- 


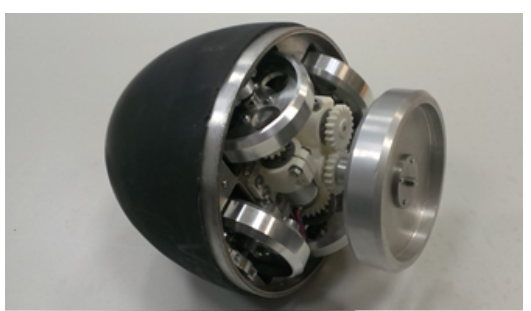

a)

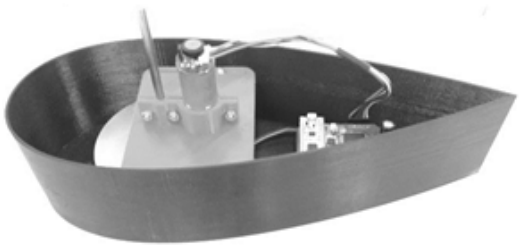

b)

Fig. 1. Propellerless underwater robot without one semi-shell (a). Propellerless aquatic robot with sharp edge (b).

entation, there is an MPU9250 sensor, which includes an accelerometer, gyroscope, and magnetometer. In the space between the rotors, the battery and the control board are located symmetrically. Data transmission for control movement can be carried out over a wired or wireless communication channel. The control system is based on a microcontroller LPC1768, control of the DC motors is carried out using the VNH3SP30 drivers.

Table 1. Values of the parameters of the robot

\begin{tabular}{lc}
\hline Parameter & Value \\
\hline Shell mass & $2.923 \mathrm{~kg}$ \\
Large rotor mass & $0.903 \mathrm{~kg}$ \\
Small rotor mass & $0.337 \mathrm{~kg}$ \\
Moment of inertia of large rotor & $7.491 \cdot 10^{-4} \mathrm{~kg} \cdot \mathrm{m}^{2}$ \\
Moment of inertia of small rotor & $0.491 \cdot 10^{-4} \mathrm{~kg} \cdot \mathrm{m}^{2}$ \\
\hline
\end{tabular}

\subsection{Design of the propellerless aquatic robot with sharp edge}

For experimental investigation the propellerless aquatic robot with internal rotor was designed. The body of the robot has the symmetric shape of a NACA 0040 airfoil profile at all heights. The robot length is $340 \mathrm{~mm}$, the width is $134 \mathrm{~mm}$, the height is $80 \mathrm{~mm}$. The foil is made on a $3 \mathrm{D}$ printer from PLA plastic, the shell thickness is $2 \mathrm{~mm}$.

The rotor with the motor is fixed inside the foil so that the center of mass of the entire system is placed as close as possible to the bottom of the robot. The rotor is made of aluminum and has an external diameter of $100 \mathrm{~mm}$, height of $12 \mathrm{~mm}$. As the actuator the gear-motor with the encoder is used. Using the encoder, we can get the angular speed of rotation of the rotor and its angular acceleration in the experiments. Inside there is placed also a control board with a STM32F303 microcontroller and a power supply. The control is realized from a personal computer or smartphone via a wireless communication channel (Bluetooth).

Table 2. Values of the parameters of the robot

\begin{tabular}{lc}
\hline Parameter & Value \\
\hline Mass of the robot & $0.905 \mathrm{~kg}$ \\
Mass of the rotor & $0.327 \mathrm{~kg}$ \\
Axial moment of inertia of robot & $0.00844 \mathrm{~kg} \cdot \mathrm{m}^{2}$ \\
Axial moment of inertia of rotor & $0.00058 \mathrm{~kg} \cdot \mathrm{m}^{2}$ \\
\hline
\end{tabular}




\section{Mathematical model of motion}

For the robots under consideration the equations of motion are written in the form of classical Kirchhoff equations: ${ }^{13}$

$$
\frac{d}{d t}\left(\frac{\partial T}{\partial \boldsymbol{V}}\right)+\boldsymbol{\Omega} \times \frac{\partial T}{\partial \boldsymbol{V}}=0, \quad \frac{d}{d t}\left(\frac{\partial T}{\partial \boldsymbol{\Omega}}\right)+\boldsymbol{\Omega} \times \frac{\partial T}{\partial \boldsymbol{\Omega}}+\boldsymbol{V} \times \frac{\partial T}{\partial \boldsymbol{V}}=0
$$

where $\boldsymbol{V}$ and $\boldsymbol{\Omega}$ are linear and angular velocity vectors of the body relative to the moving coordinate system attached to the body; $T$ is the total kinetic energy of the system, which consists of the kinetic energy of the foil, the kinetic energy of the fluid, and the kinetic energy of the rotors.

For the propellerless underwater robot the system of equations describing motion has the form:

$$
\begin{gathered}
\mathbf{C} \dot{\boldsymbol{V}}+\mathbf{B} \dot{\boldsymbol{\Omega}}=(\mathbf{C} \boldsymbol{V}+\mathbf{B} \boldsymbol{\Omega}) \times \boldsymbol{\Omega}, \\
\mathbf{B}^{T} \dot{\boldsymbol{V}}+\mathbf{I} \dot{\boldsymbol{\Omega}}+\dot{\boldsymbol{K}}(t)=\left(\mathbf{B}^{T} \boldsymbol{V}+\mathbf{I} \boldsymbol{\Omega}+\boldsymbol{K}(t)\right) \times \boldsymbol{\Omega}+(\mathbf{C} \boldsymbol{V}+\mathbf{B} \boldsymbol{\Omega}) \times \boldsymbol{V}=0 \\
\dot{\boldsymbol{\alpha}}=\boldsymbol{\alpha} \times \boldsymbol{\Omega}, \quad \begin{array}{l}
\dot{\boldsymbol{\beta}}=\boldsymbol{\beta} \times \boldsymbol{\Omega}, \quad \dot{\boldsymbol{\gamma}}=\gamma \times \boldsymbol{\Omega}, \\
\dot{\boldsymbol{r}}=\mathbf{Q}^{T} \boldsymbol{V} .
\end{array}
\end{gathered}
$$

where matrix $\mathbf{I}$ takes into account the moments of inertia of the shell and rotors, the added moment of inertia; matrix B takes into account location of the system's center of mass; matrix $\mathbf{C}$ takes into account added masses; $\boldsymbol{r}=(x, y, z)$ are coordinates of the geometric center of the shell in a fixed coordinate system; $\boldsymbol{\alpha}, \boldsymbol{\beta}, \boldsymbol{\gamma}$ are unit vectors of fixed axes referred to the moving axes; $\mathbf{Q}=(\boldsymbol{\alpha}, \boldsymbol{\beta}, \boldsymbol{\gamma})$ is the matrix of transition from the fixed coordinate system to the moving one; $\boldsymbol{K}(t)$ is the gyrostatic momentum vector, which is the control action.

The added masses and the added moment of inertia for the shape of ellipsoid of revolution were calculated using reference materials. A detailed derivation of the equations of motion can be found in. ${ }^{14}$

For the propellerless aquatic robot with sharp edge a model of plane-parallel motion is considered, which takes into account the resistance of the liquid. The system of equations describing motion has the form:

$$
\begin{gathered}
\left(m+\lambda_{11}\right) \dot{v}_{1}=\left(m+\lambda_{22}\right) v_{2} \omega+\lambda_{23} \omega^{2}-c_{1} v_{1}\left|v_{1}\right|, \\
\left(m+\lambda_{22}\right) \dot{v}_{2}+\lambda_{23} \dot{\omega}=-\left(m+\lambda_{11}\right) v_{1} \omega-c_{2} v_{2}\left|v_{2}\right|, \\
\lambda_{23, l} \dot{v}_{2}+\left(I+\lambda_{33}\right) \dot{\omega}=\left(\lambda_{11}-\lambda_{22}\right) v_{1} v_{2}-\lambda_{23, r} v_{1} \omega-c_{3} \omega|\omega|-\dot{k}(t), \\
\dot{x}=v_{1} \cos \alpha-v_{2} \sin \alpha, \quad \dot{y}=v_{1} \sin \alpha+v_{2} \cos \alpha, \quad \dot{\alpha}=\omega .
\end{gathered}
$$

where $x$ and $y$ are the center of mass coordinates; $m$ - mass of the robot; $\alpha$ - the angle that determines the robot's orientation; $v_{1}, v_{2}$ are the projections of the linear velocity vector on the axes of the moving coordinate system, $\omega$ is the angular velocity of the foil; $c_{1}, c_{2}, c_{3}$ are the drag coefficients; $\lambda_{11}, \lambda_{22}$ are the added masses, $\lambda_{33}$ is the added moment of inertia, and $\lambda_{23}$ is the coefficient due to disalignment of the buoyancy center and the center of mass; $k(t)$ is the gyrostatic moment, which is the control action.

In equations (3) coefficients $\lambda_{i j}$ and $c_{i}$ were determined using the numerical solution of the Navier-Stokes equations using the experimental data obtained by the symmetric control action. A detailed derivation of the equations of motion can be found in. ${ }^{15}$ 


\section{Experimental investigations}

\subsection{Experiments with propellerless underwater robot}

Three series of experiments were conducted with a screwless underwater robot to determine the motion patterns with various control actions. In the each experiments the rotors were accelerated to a maximum speed of $590 \mathrm{rpm}$, which was maintained for 3 seconds. The experiments were conducted in a pool of $3 \times 1.5 \times 1.5$ meters. The trajectories were tracked using the Vicon motion capture system.

1. Rotation of a pair of large rotors. The gyrostatic momentum vector is $K=$ $\left(2 i_{1} \omega_{\max }, 0,0\right)$. In the Figure $2 \mathrm{a}$, the solid line shows the theoretical trajectory of the movement, and the dashed line shows the experimental trajectories.

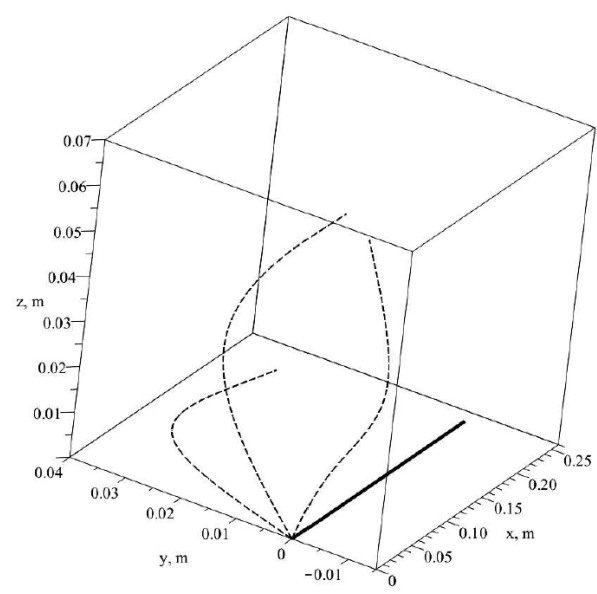

a)

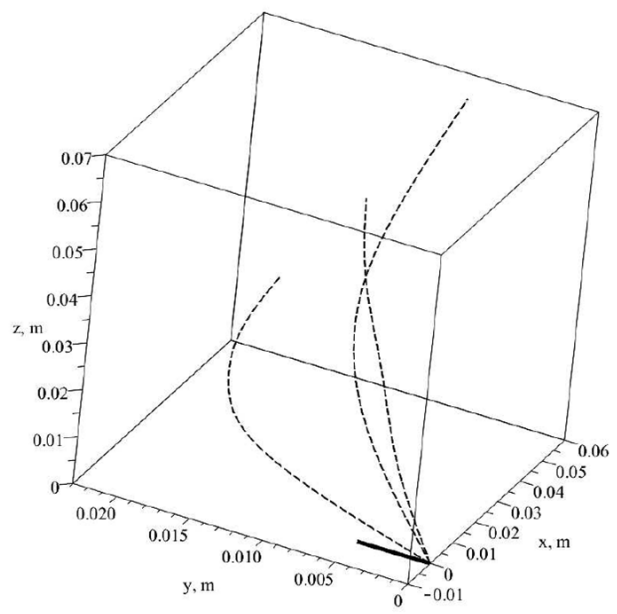

b)

Fig. 2. Theoretical and experimental trajectory of moving of propellerless underwater robot for $K=$ $\left(2 i_{1} \omega_{\max }, 0,0\right)(\mathrm{a})$ and $K=\left(0,2 i_{2} \omega_{\max }, 0\right)(\mathrm{b})$

A comparison of the values of the robot's movement and the angles that determine the robot's orientation is shown in the table 3 .

Table 3. A comparison of experimental and modelling results for $K=\left(2 i_{1} \omega_{\max }, 0,0\right)$

\begin{tabular}{cccccccc}
\hline & $\Delta x$, & $\Delta y$, & $\Delta z$, & $\left|\boldsymbol{r}_{t}\right|$, & $\Delta \theta$ & $\Delta \psi$ & $\Delta \varphi$ \\
\hline Modelling & 0.275 & 0 & 0 & 0.275 & $0^{\circ}$ & $0^{\circ}$ & $738.2^{\circ}$ \\
Experiment & 0.115 & 0.010 & 0.055 & 0.128 & $4^{\circ}$ & $10^{\circ}$ & $121^{\circ}$ \\
\hline
\end{tabular}

2. Rotation of a pair of small rotors. The gyrostatic momentum vector is $K=$ $\left(0,2 i_{2} \omega_{\max }, 0\right)$. In the Figure $2 \mathrm{~b}$, the solid line shows the theoretical trajectory of the movement, and the dashed line shows the experimental trajectories.

A comparison of the values of the robot's movement and the angles that determine the robot's orientation is shown in the table 4.

3.Rotation of a pair of small rotors and a pair of large rotors. The gyrostatic momentum vector is $K=\left(2 i_{1} \omega_{\max }, 2 i_{2} \omega_{\max }, 0\right)$. In the Figure 3 , the solid line shows the theoretical trajectory of the movement, and the dashed line shows the experimental trajectories.

A comparison of the values of the robot's movement and the angles that determine the 
Table 4. A comparison of experimental and modelling results for $K=\left(0,2 i_{2} \omega_{\max }, 0\right)$

\begin{tabular}{cccccccc}
\hline & $\Delta x$, & $\Delta y$, & $\Delta z$, & $\left|\boldsymbol{r}_{t}\right|$, & $\Delta \theta$ & $\Delta \psi$ & $\Delta \varphi$ \\
Modelling & 0 & 0.005 & 0 & 0.005 & $35^{\circ}$ & $0^{\circ}$ & $0^{\circ}$ \\
Experiment & 0.054 & 0.008 & 0.068 & 0.087 & $61^{\circ}$ & $62^{\circ}$ & $10^{\circ}$ \\
\hline
\end{tabular}

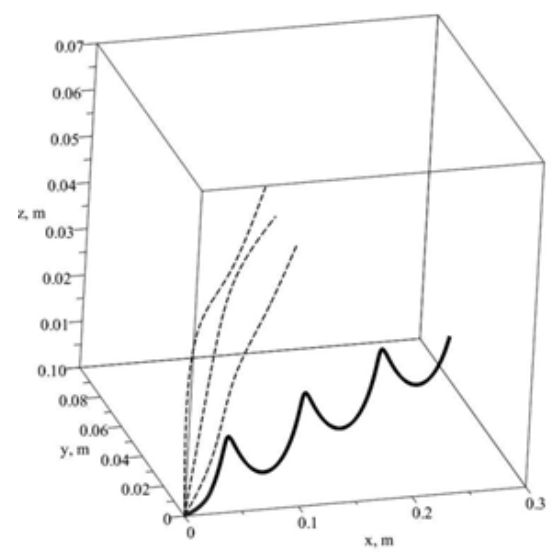

Fig. 3. Theoretical and experimental trajectory of moving of propellerless underwater robot for $K=$ $\left(2 i_{1} \omega_{\max }, 2 i_{2} \omega_{\max }, 0\right)$

robot's orientation is shown in the table 5 .

Table 5. A comparison of experimental and modelling results for $K=\left(2 i_{1} \omega_{\max }, 2 i_{2} \omega_{\max }, 0\right)$

\begin{tabular}{cccccccc}
\hline & $\Delta x$, & $\Delta y$, & $\Delta z$, & $\left|\boldsymbol{r}_{t}\right|$, & $\Delta \theta$ & $\Delta \psi$ & $\Delta \varphi$ \\
Modelling & 0.275 & 0 & 0 & 0.275 & $35^{\circ}$ & $0^{\circ}$ & $738.2^{\circ}$ \\
Experiment & 0.106 & 0.050 & 0.053 & 0.189 & $17^{\circ}$ & $92^{\circ}$ & $51^{\circ}$ \\
\hline
\end{tabular}

Conclusions:

- The controlled movement of the robot continues as long as the accelerated rotation of the rotors is provided.

- Acceleration of the rotors to the maximum speed takes a certain time, which is not taken into account in the theoretical model but has influence on the trajectory of the robot.

- The theoretical model is developed using an model with ideal viscosity that also explains discrepancies between the theoretical and real trajectory of movement.

- The movement of the robot is accompanied by the formation of vortex structures. It is difficult to provide a vortex-free movement using rotors, as the theory requires.

- The model describes movement qualitatively, but quantitative agreement is affected by the accuracy of determining a large number of parameters. Movement is possible, but its effectiveness is not high.

Based on the conclusions made above, the following points were taken into account in the work on the propellerless aquatic robot with a sharp edge:

- A plane-parallel motion on the surface of a fluid is considered.

- Using the shape of a shell with a sharp edge, the formating vortices do not hinder the progress, but help it. 
- The motion model takes into account the accelerated rotation of the rotor, as well as the viscous friction of the fluid

- The rotation of the rotor occurs according to the periodic law.

\subsection{Experiments with propellerless aquatic robot with a sharp edge}

For the presented robot model, a simplified finite-dimensional motion model was developed, which allows one to determine the shape of the motion paths under various control actions. To test the theoretical model, experimental investigations were conducted in a $2 \times 1.2$ meter pool. The trajectories were tracked using the Vicon motion capture system.

We define the function of the rotor's rotation, $\Omega(t)$, by a piecewise continuous periodic function (see Figure 4).

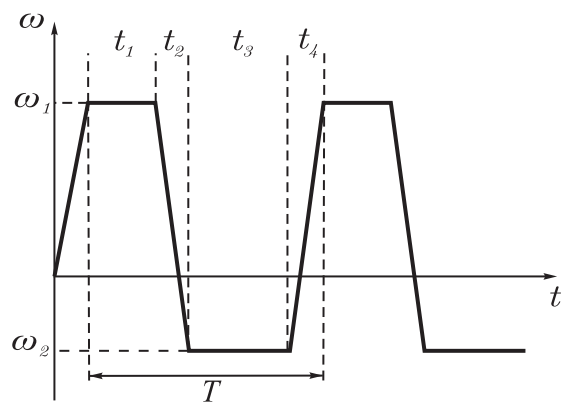

Fig. 4. A graph of the angular rotational speed of the rotor versus time in general form. $T$ is the period of control action, $t_{1}, t_{3}$ define time intervals with constant angular rotational speeds of the rotor, $\omega_{1}$ and $\omega_{2}$, respectively, and $t_{2}, t_{4}$ are the intervals of uniformly accelerated rotation of the rotor

Below we consider the results of modelling and experimental investigations for straightline motion, motion in a circle and motion in a complex trajectory.

To move in a straight line it is necessary that the control action be of symmetric form, so that $t_{2}=t_{4} \approx 0.1 \mathrm{~s}$., $t_{1}=t_{3}=0.5\left(T-2 t_{2}\right)$. Several experiments were carried out these conditions $\omega_{1}=\omega_{\max }, \omega_{2}=-\omega_{\max }$, the period of control action $T$ was changed. The results of the experiments are shown in accordance with Figure 5.

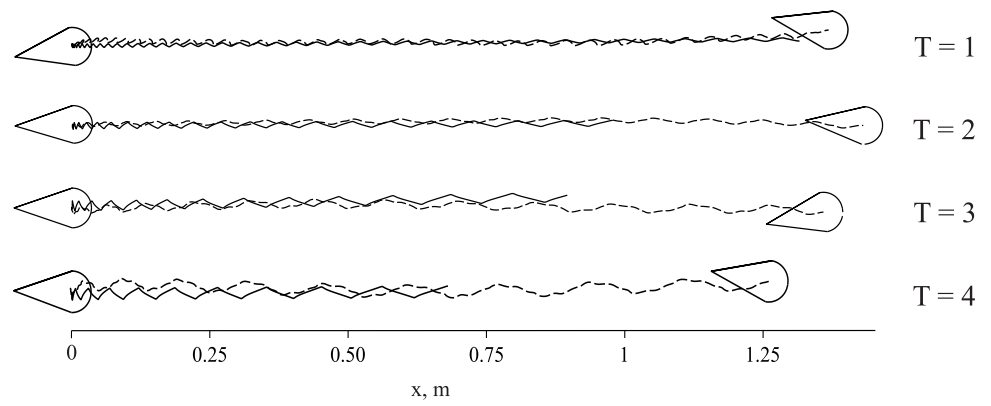

Fig. 5. Trajectories of the robot for $\omega_{1}=\omega_{\max }, \omega_{2}=-\omega_{\max }$ and different control actions. The solid lines indicate the trajectories obtained from the results of numerical simulation, and the dashed lines denote experimental trajectories.

While maintaining the conditions $t_{2}=t_{4}, t_{1}=t_{3}$ and for $\omega_{1}-\omega_{2}=$ const and $\omega_{1}-\omega_{2} \neq$ $2 \omega_{\max }$ robot also moves in a straight line. At the beginning of the movement, the robot turns at a certain angle, which depends on the value $\omega_{1}-\omega_{2}$. An example of control actions and corresponding motion paths is shown in the Figure 6. 


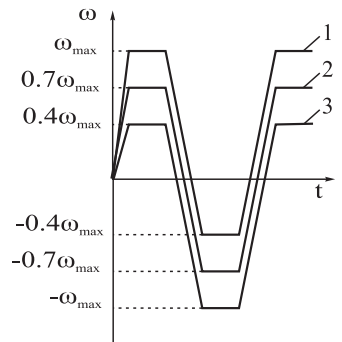

a)

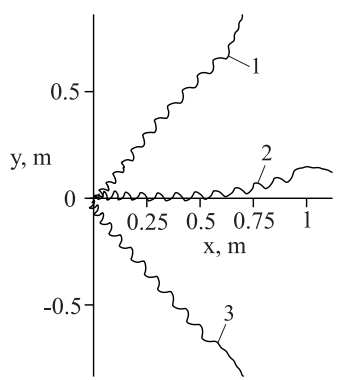

b)

Fig. 6. Asymmetric control actions at $\omega_{1}-\omega_{2}=$ const (a) and corresponding trajectories of the aquatic robot (b)

For $t_{1} \neq t_{3}$ or $t_{2} \neq t_{4}$ robot moves along a circle. Two experiments were performed with the following parameters: $t_{1} \neq t_{3}, t_{2}=t_{4}$; results of experiment are shown in Figure $7 \mathrm{a}$. $t_{1}=t_{3}, t_{2} \neq t_{4}$; results of experiment are shown in Figure $7 \mathrm{~b}$.

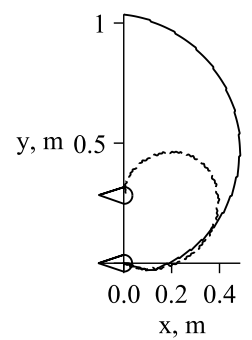

a)

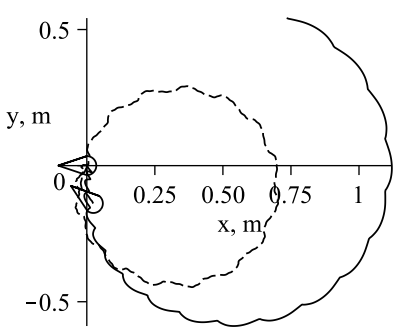

b)

Fig. 7. Trajectory of propellerless aquatic robot with sharp edge along a circle for $t_{1} \neq t_{3}, t_{2}=t_{4}(\mathrm{a})$ and $t_{1}=t_{3}, t_{2} \neq t_{4}(\mathrm{~b})$. The solid lines indicate the trajectories obtained from the results of numerical simulation, and the dashed lines denote experimental trajectories.

Combining the considered control actions, it is possible to realize movement along complex trajectories. An example of movement along this trajectory is shown in the Figure 8.

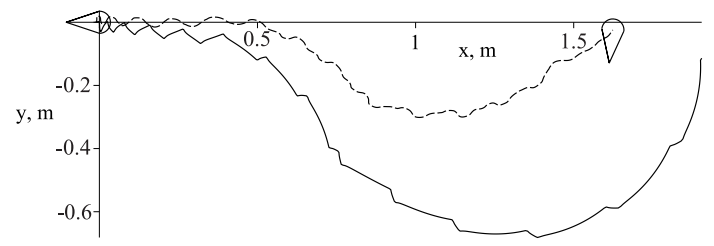

Fig. 8. Complex trajectory of the robot. The solid lines indicate the trajectories obtained from the results of numerical simulation, and the dashed lines denote experimental trajectories.

We can conclude that the theoretical model qualitatively describes the robot's movement correctly.

\section{Conclusions}

It is experimentally shown that the movement of propellerless aquatic robots controlled by the rotation of internal rotors is possible. A theoretical model based on equations of motion in an ideal fluid does not describe motion qualitative well enough. For a good qualitative and quantitative agreement, it is necessary to supplement the motion models with viscous coeffi- 
cients, take into account the fluid circulation, and more accurately determine the coefficients of the added masses.

\section{Acknowledgments}

The work of Yu. L. Karavaev, A. V. Klekovkin, V.A. Tenenev was carried out within the framework of the state assignment of the Ministry of Education and Science of Russia (FZZN-2020-0011). The work of E. V. Vetchanin was carried out within the framework of the state assignment of the Ministry of Education and Science of Russia (FEWS-2020-0009). The work of I. S. Mamaev and was supported by the RFBR Grant No. 18-08-00995-a and by the RFBR Grant No. 18-29-10050-mk. The experimental investigations were carried out using the equipment of the Common Use Center of the Udmurt State University.

\section{References}

1. Chernousko F. L., Motion of a body in a fluid due to attached-link oscillations, Dokl. Akad. Nauk, vol. 431, no. 1, pp. 46-49 [Dokl. Phys., 2010, vol. 55, no. 3, pp. 138-141].

2. Chernousko F. L., Bolotnik N. N., Figurina T. Y., Optimal control of vibrationally excited locomotion systems, Regular and Chaotic Dynamics, 2013, vol. 18, no. 1-2, pp. 85-99

3. Chernousko F. L., Optimal two-dimensional motions of a body controlled by a moving internal mass, Multibody System Dynamics, 2019, vol. 46, no. 4, pp. 381-398.

4. Vorochaeva (Volkova) L. Y., Jatsun S. F., Control of the three-mass robot moving in the liquid environment, Rus. J. Nonlin. Dyn., 2011, Vol. 7, No. 4, pp. 845-857

5. Xu J., Fang H., Improving performance: recent progress on vibration-driven locomotion systems. Nonlinear Dyn, 2019, vol. 98, pp. 2651-2669 https://doi.org/10.1007/s11071-019-04982-y

6. Pollard B., Tallapragada P., An aquatic robot propelled by an internal rotor, IEEE/ASME Transactions on Mechatronics, 2016, Vol. 22, no. 2, pp. 931-939.

7. Nunuparov A., Becker F., Bolotnik N., Zeidis I., Zimmermann K. Dynamics and motion control of a capsule robot with an opposing spring Archive of Applied Mechanics, 2019, vol. 89. no. 10, pp. 2193-2208.

8. Kozlov V. V., Ramodanov S. M., The Motion of a variable body in an ideal fluid, J. Appl. Math. Mech., 2001, vol. 65, no. 4, pp. 579-587; see also: Prikl. Mat. Mekh., 2001, vol. 65, no. 4, pp. 592-601.

9. Kozlov V. V., Onishchenko D. A., The Motion in a perfect fluid of a body containing a moving point mass, J. Appl. Math. Mech., 2003, vol. 67, no. 4, pp. 553-564; see also: Prikl. Mat. Mekh., 2003, vol. 67, no. 4, pp. 620-633.

10. Vetchanin E. V., Kilin A. A., Controlled Motion of a Rigid Body with Internal Mechanisms in an Ideal Incompressible Fluid, Proceedings of the Steklov Institute of Mathematics, 2016, vol. 295, pp. 302-332

11. Klenov A. I., Kilin A. A., Influence of Vortex Structures on the Controlled Motion of an Abovewater Screwless Robot, Regular and Chaotic Dynamics, 2016, vol. 21, no. 7-8, pp. 927-938

12. Tahmasian S., Jafaryzad A., Bulzoni N. L., Staples A.E., Dynamic Analysis and Design Optimization of a Drag-Based Vibratory Swimmer Fluids, 2020, vol. 5, no. 1, p. 18.

13. Kirchhoff G. R. Ueber die Bewegung eines Rotationskörpers in einer Flüssigkeit // J. für Math., $1869,223-273$

14. Karavaev Y.L., Kilin A. A., Klekovkin A. V., Experimental Investigations of the Controlled Motion of a Screwless Underwater Robot, Regular and Chaotic Dynamics, 2016, vol. 21, no. 7-8, pp. 918-926

15. Karavaev Y. L., Klekovkin A. V., Mamaev I. S., Tenenev V. A., Vetchanin E. V., A Simple Physical Model for Control of an Propellerless Aquatic Robot, Mechanical Systems and Signal Processing, 2020, unpublished 